\title{
“知识、能力、素养”融合的无机化学课程新教学模式建设
}

张霞 ${ }^{*}$, 桑晓光, 王锦霞, 孟皓

东北大学理学院化学系, 沈阳 110819

摘要: 东北大学无机化学课程改革立足课程教学的育人功能, 将知识的学习、能力的培养与人才素养的提升紧密结 合。教学内容体系建设上, 以 “坚、宽、深、交” 知识结构为立足点, 拓宽课堂教学体系, 注重交叉科学知识, 突 出理论联系实际, 融入课程思政元素; 教学模式上, 实践 “对分课堂”, 结合PBL案例教学, 转换教师与学生的课 堂教学地位, 激发学生主动式自主学习能力; 引入无机化学双语课堂, 开阔学生的国际化视野; 发挥无机化学MOOC 课堂的在线学习功能, 开展线上线下混合式教学; 创新无机化学实验课程考核方式, 确保实验课程的学习和能力培 养目标的达成。总的说来, 通过完善课程教学体系、创新教学模式, 有效地提升课程教学效果, 为东北大学理工科 学生打下良好的化学基础, 培养化学思维习惯, 践行 “三全育人” 教育理念。

关键词：课程育人；无机化学课程改革；课程思政；专业素养；能力培养

中图分类号: G64; O6

\section{Construction of New Teaching Mode of Inorganic Chemistry by Integrating Knowledge, Ability and Quality}

\author{
Xia Zhang ${ }^{*}$, Xiaoguang Sang, Jinxia Wang, Hao Meng \\ Department of Chemistry, College of Sciences, Northeastern University, Shenyang 110819, China.
}

\begin{abstract}
In Northeastern University, the reform of inorganic chemistry course has been focused on the educational function of the classroom teaching, which tightly combines the knowledge learning, ability cultivation and quality improvement. In the construction of teaching system, guided by the idea of "firm, broad, deep and interactive", the classroom teaching contents are broadened, the inter-disciplinary scientific knowledge is introduced, the theory learning is integrated with practice, and the ideological and political elements are also combined. With respect to the teaching method, the split classroom is combined with the PBL mode, as a result, roles of teachers and students in the classroom are exchanged, and the students' active self-learning ability is promoted. Furthermore, the bilingual classroom is also employed, which significantly broaden the students' international vision. The applications of MOOC of inorganic chemistry endow the abundant online learning function, based on which the hybrid teaching mode is also practiced. In addition, the course of inorganic experiments is also improved by reforming the testing method in order to ensure the training objectives involving learning new knowledge and ability. Generally speaking, through improving the teaching system and innovating the teaching mode, the teaching effects of the course have been effectively enhanced, which lay a good chemistry foundation for the students major in science and engineering, and cultivate the chemical thinking habit, and practice the idea of "Three" education.
\end{abstract}

Key Words: Curriculum education; Reform of inorganic chemistry course; Course ideology and politics;

Professional quality; Ability development

收稿: 2021-07-25; 录用: 2021-07-28; 网络发表: 2021-09-03

“通讯作者, Email: xzhang@mail.neu.edu.cn

基金资助: 东北大学理学院教育教学改革项目立项资助(2021年) 
培养高素质复合型人才是高校教育教学的主要工作目标。习近平同志在全国教育大会上指出, 要努力构建德智体美劳全面培养的教育体系, 要把立德树人融入思想道德教育、文化知识教育、社 会实践教育各环节。在高校, 课堂是促进学生全面发展最重要的阵地, 课堂教学改革是现阶段高等 教育教学改革的关键主题。在课堂教学环节, 强化学生的知识积累、能力培养、价值引领以及人格 塑造, 让课堂真正彰显育人为本的价值导向, 让教师肩负起立德树人的使命担当, 让学生在有质量 的高品质课堂中塑造品格。高品质课堂的建设将学生的主体能动性与教师的主导作用以及教学内容 的育人功能三要素协同统一, 高质量地促进每一名学生的健康成长 ${ }^{[1,2]}$ 。

无机化学课程是东北大学理工科的公共基础课。开设无机化学课程的大类专业班级有 37 个班, 约 1100 名学生。无机化学课程学习对于培养东北大学理工科学生的化学思维素质发挥重要作用。近 年来, 无机化学课程改革立足课堂教学的育人功能, 将知识的学习与能力的培养和人才素养的提升 紧密结合。课堂教学内容增加无机化学前沿知识, 强化理论联系实际, 与专业知识相衔接; 在教学 模式上, 结合 PBL 实例教学, 建设无机化学 “对分课堂”, 调动学生的自主学习兴趣, 培养学生的 自主学习能力。引入无机化学双语课堂, 发挥英文教学在无机化学课堂教学中的优势。随着无机化 学 MOOC 课程获批国家级一流课程, 结合在线课程的教学优势, 开展线上线下混合式教学新样态。

总的说来, 无机化学课程的改革与教学实践主要体现在以下四个方面。

\section{1 特色无机化学教学内容建设与实践, 提升专业素养}

针对大类培养模式, 调整原工科无机化学教学内容, 重新组织课堂教学体系。作为一门公共基 础课, 无机化学教学内容设计充分重视各大类专业的培养目标与培养需求, 在教学内容的组织上, 夯 实无机化学基础理论知识同时, 加强应用基础知识解决实际问题的实训。我们的做法是在完成教学 大纲基本要求的基础上, 允许教师根据学生专业特色, 增加授课内容, 激发学生学习兴趣。

每一位主讲教师在授课过程中都会有意识地结合学生的专业及教师的科研经历, 灵活地开展延 伸教学, 或联系生产实践, 或与专业科研相关联, 构建特色教学内容。表 1 是主讲教师在无机化学 教学大纲基础之上, 在课堂教学中设计的部分具有专业特色的教学内容。通过具有专业特色的延伸 教学内容, 在提升学生兴趣的同时, 将基础课程理论知识学习与专业学习以及生产实践相关联, 发 挥基础理论课程的教学功能。在专业知识传授过程中, 融合思政元素, 更加注重突出中国专家学者 的理论贡献以及严谨的科学精神, 体现 “三全育人” 教育理念。

\section{PBL 教学及 “对分课堂” 教学模式实践, 强调主动式学习}

PBL (Problem-Based Learning) 教学是以真实案例为背景提出问题, 激发和引导学生以小组为单 位自主探究、合作学习解决问题的学习方式。PBL 教学模式强调以问题为核心, 以学生为中心, 注 重合作学习、评价多元化的特点, 有效培养了学生自主学习与终身学习的能力, 加强了理论与实践 的结合, 从而提高了学生应用知识的能力, 培养了学生团队合作能力 ${ }^{[3-5]}$ 。

无机化学课程 PBL 教学设计遵循以下原则: 循序渐进的原则合理设置问题梯度; 有效的问题情 境, 具有真实性、目标性和简洁性; 问题的提出具有明确的目标性, 使学生能准确把握探索的方 向。无机化学课程的授课对象为大一新生, 学生普遍自我学习意识较差, 学习能力较弱, 因此内容 过于庞大、综合性较强的 PBL 实例学生解决起来会比较困难, 可能会引起学生的反感, 压制学生学 习积极性。因此, 我们针对无机化学课程特点, 在课程教学不同阶段, 设计了内容、难度、综合性 不同的 PBL 教学课例, 分阶段引导学生实施。

在学期初, 教师有意识地引导学生关注 PBL 教学新模式, 讲授如何查阅文献, 并结合无机化学 的前沿知识给学生布置了第一个 PBL 课例, 学生通过百度或者查阅杂志、新闻报道可以完成。并在 随后的课堂上随机挑选同学汇报课例作业, 教师适当通过问题引导全体同学参与讨论过程, 掌握如 
大学 化 学 Univ. Chem. 2021, 36(11), 2107081 (3 of 6)

表 1 专业特色无机化学教学内容举例

\begin{tabular}{|c|c|c|}
\hline 章节 & 专业特色教学内容 & 教学内容属性 \\
\hline \multirow[t]{2}{*}{ 第 1 章 } & 医学上的溶血现象及等渗、高渗、低渗溶液原理 & 理论联系实际 \\
\hline & 超临界干燥法在样品处理中应用 & 理论联系实际 \\
\hline \multirow[t]{2}{*}{ 第 2,3 章 } & 举例说明工业设计反应需要考虑的因素 & 理论联系实际 \\
\hline & 微观反应动力学-飞秒化学 & 前沿知识 \\
\hline \multirow[t]{3}{*}{ 第 4,5 章 } & 未知混合离子的分离分析方法 & 理论联系实际 \\
\hline & 针对实际反应的缓冲体系选择及应用 & 理论联系实际 \\
\hline & 举例说明沉淀平衡在金属离子分离分析中的应用 & 理论联系实际 \\
\hline \multirow[t]{5}{*}{ 第 6 章 } & 湿法冶炼金单质的氨化物法 & 理论联系实际 \\
\hline & 锂电池的电化学反应及电池工作原理 & 理论联系实际 \\
\hline & 化学电池的发展及前景 & 前沿知识 \\
\hline & 光解水制氢的原理及发展前景 & 前沿知识 \\
\hline & 2019 年诺贝尔化学奖的获得者及学术贡献 & 前沿知识 \\
\hline \multirow[t]{3}{*}{ 第 7 章 } & 徐光宪院士的 $n+0.71$ 和 $n+0.41$ 规则 & 课程思政 \\
\hline & 唐敖庆院士的杂化轨道成键能力计算 & 课程思政 \\
\hline & 扫描隧道显微镜原子观察和原子操纵 & 前沿知识 \\
\hline \multirow[t]{2}{*}{ 第 8 章 } & 水分子结构的特异性 & 理论联系实际 \\
\hline & 计算化学 & 前沿知识 \\
\hline \multirow[t]{2}{*}{ 第 9 章 } & $\mathrm{X}$ 射线衍射分析方法 & 理论联系实际 \\
\hline & 神奇的碳家族-富勒烯, 碳纳米管, 石墨烯 & 前沿知识 \\
\hline \multirow[t]{2}{*}{ 第 10 章 } & 神奇的金属有机骨架化合物(MOFs) & 前沿知识 \\
\hline & 核磁共振影像照影剂 & 前沿知识 \\
\hline
\end{tabular}

何更好地完成 PBL 教学任务。在学期中, 结合无机化学反应理论基础知识, 给出了中等难度的 PBL 教学课例, 学生需要查阅专业书籍或者专业文献才能给出作业答案。采取在课堂上随机挑选同学汇 报作业方案, 教师与同学共同点评, 引导学生如何确定课题研究目标, 并利用学校数据库资源查找 文献。在此阶段学生基本了解了学校专业数据库的使用, 以及如何利用关键词组合查找所需的文献 内容, 并加以归纳总结, 回答 PBL 作业问题。在学期末, 结合无机化学课程元素化学部分的学习特 点和学习要求, 针对我国稀土元素开发利用现状, 给出综合性较强的 PBL 大作业。学生需要综合查 阅文献资料, 给出结论并自行提出解决方案。要求学生依据文献调研结果, 给出 2000-5000 字的 PBL 作业方案。收集上来的结果表明, 有 $70 \%$ 的同学交 PBL 作业的字数超过 5000 字, 有 $20 \%$ 同学的 PBL 建设方案详细、全面, 并且有自己的建设性意见, 较好地完成了 PBL 教学任务, 达到了 PBL 教学的 能力培养目标。

在课堂教学过程中, 围绕 PBL 教学课例的任务结果, 采取对分课堂的教学方式 ${ }^{[6,7]}$ 。分配一半课 堂时间给教师讲解, 另一半给学生讨论, 确保师生对教与学的 “责任分担”。教师在讲解重点、难点 知识以后, 采取多种形式让学生来参与课堂教学, 实现课堂教与学完美互动, 教师与学生共同完成 教学内容。提升课堂教学环节对于学生能力的培养, 真正做到学以致用。利用无机化学 MOOC 的在 线教学优势, 开展线上线下混合式教学, 为我们省出了更多的课堂时间, 开展多种课堂教学模式、 翻转课堂, 课程教学的灵活性和主动性得到了很好的体现。 


\section{3 无机化学双语教学内容精心设计及课堂教学组织, 开拓学生国际视野}

在现代科学领域, 专业英语被应用的场合越来越多。国际专业学术会议和科技资料 $85 \%$ 用英文 出版, 交流方式也从过去的阅读和翻译转变为听、说、读、写、译。因此, 能够熟练运用英语获取 最新科研动态和与外国同行专家交流的专业技术人才受到人们的欢迎。随着学科交叉不断加强, 化 学专业英语在冶金、材料、矿物、环境等专业文献中出现的频率也越来越高。因此, 采用双语模式 教学对于理工科专业的学生来说, 既掌握了无机化学专业知识, 又熟悉化学专业词汇, 对于学生后 续专业学习是非常有好处的。

我们的两位主讲教师参加了东北大学与美国北卡罗来纳州立大学的教师教学培训计划, 将美国 主动式学习的英文课堂模式融入无机化学课堂教学。为了达到最优的课堂教学效果, 我们从众多的 外文书中优选与课程的中文书体系、内容相似的英文教材, 中英文教材对照使用。教师在每学期开 课前做出详细的教学日历, 包括每一节课的教学内容, 有计划地安排学生预习、复习英文教材的相 关内容。课堂上教师教学语言以英文为主, 强迫学生熟悉英文的教学环境。在期中期末考核中增加 英文试题的比例, 这样在考查学生专业知识的同时, 对于学生的专业英语水平进行综合考量。我们 期望通过严格的英文教学过程, 让我们的学生与国际社会接轨, 开阔视野, 同时为学生将来的出国 交流与进修建立良好的专业基础。

目前我们的无机化学双语课程已经用于教学实践的教学资源, 包括两本英文教材: (1) 《General Chemistry》, by Ralph H. Petrucci, et al. 高等教育出版社, 2004 年; (2) 《General Chemistry》, by Ming Zhao, 高等教育出版社, 2014 年; 此外, 已经建设课程全部内容英文 PPT, 英文教学日历, 期 中、期末考试英文题库等。

\section{4 无机化学实验课程建设, 提升实验课程育人功能}

在不断更新无机化学实验项目基础上, 我们着重进行了实验课程考核办法改革, 力求提升学生 的实验课程学习兴趣, 同时能够客观地评价学生对于实验操作技术的掌握程度。

传统的无机化学实验课考核方法以平时成绩为准, 只强调了单个实验, 没有最后的期末考核环 节。为了加深学生对实验课的理解, 确保实验教学效果, 增加了期末考核环节。考核由笔试和操作 两种形式构成。笔试以理论卷的形式考查学生对知识的掌握情况。操作以现场操作形式考核, 学生 随机抽签, 根据抽到的实验题目, 独立完成实验。根据学生的语言表达、思维逻辑、学生在实验操 作过程中仪器使用的熟练程度及实验顺序安排的合理性, 监考教师根据评分标准现场打分。

部分期末无机化学实验操作考试的试题如下:

(自行设计实验, 写出实验目的、实验用品、实验原理、实验步骤、结果与讨论)

1) 未知溶液的鉴定。鉴定出未知溶液中的两种阳离子。

范围: $\mathrm{Cr}^{3+} 、 \mathrm{Fe}^{3+} 、 \mathrm{Co}^{2+} 、 \mathrm{Ni}^{2+} 、 \mathrm{Cu}^{2+}$

2) 比较 $\mathrm{Fe}(\mathrm{SCN})_{3}$ 和 $\mathrm{FeF}_{6}^{3-}$ 两种配合物的稳定性。

3) 比较 $\mathrm{AgCl} 、 \mathrm{AgBr}$ 和 $\mathrm{AgCl}$ 溶解度的大小。

4) 证明酸碱性对 $\mathrm{KMnO}_{4}$ 氧化性的影响。

5) 桌上有一未知溶液, 可能为 $\mathrm{Fe}^{3+} 、 \mathrm{Co}^{2+}$ 中的一种或者两种, 如何鉴别? 写出鉴定方法和鉴定 结果。

6) 利用比色法检测未知溶液中的 $\mathrm{Fe}^{3+}$ 浓度。

$\left(50 \mathrm{~mL}\right.$ 容量瓶中为实验室提供的 $5 \times 10^{-4} \mathrm{~mol} \cdot \mathrm{L}^{-1}$ 的硫酸铁铵溶液)

7) 测定得到下面反应的活化能。

$2 \mathrm{Fe}\left(\mathrm{NO}_{3}\right)_{3}+2 \mathrm{KI}=2 \mathrm{Fe}\left(\mathrm{NO}_{3}\right)_{2}+\mathrm{I}_{2}+2 \mathrm{KNO}_{3}$

此反应的速率方程式为:

$$
v=k\left[c\left(\mathrm{Fe}\left(\mathrm{NO}_{3}\right)_{3}\right)\right][c(\mathrm{KI})]^{2}
$$


在实验课程考核方案设计过程中, 我们重点落实了无机化学实验课程评分标准规范化。由于实 验课程学生人数众多, 多名教师共同完成实验教学任务。为了确保实验评价的客观性, 我们将实验 教学每一个环节中的各项内容, 例如实验报告中的实验目的、实验原理、实验步骤、结果与讨论、 习题等各占多少比重进行了更详细的规定, 制定了无机化学实验评分标准, 所有指导教师参照执行。

通过加强实验课程的考核办法, 督促学生高质量地的完成实验教学内容, 确保实现实验教学对 于学生能力的培养和素质提升功能。

\section{5 结语}

总的说来, 通过教学内容的重组、PBL 教学和课程思政元素的融合以及 “对分课堂” 教学模式 建设, 无机化学的课程教学效果在不断提升。课程改革实践的直接受益者是学生。无机化学课程 的教改成果主要应用于东北大学理工科的无机化学和大学化学公共基础课程学习。通过近年来无 机化学课程的不断改革、实践、完善, 在东北大学理工科学生的化学知识学习、储备, 思维习惯 培养及分析解决问题能力提升方面发挥重要作用。一是体现在学生的课堂参与积极性明显提高, 课 程教学受到了学生的普遍欢迎, 无机化学课程的教师评分基本保持在 90 分以上。主讲教师张霞教授 为省教学名师, 两次入选东北大学我最喜爱教师提名奖, 无机化学课程获评为国家级和省级一流本 科课程; 另一方面, 体现在学生课程成绩的普遍提升, 最直观的体现是虽然课程考核的灵活度不断 提高、过程考核不断加强, 但是课程总评成绩的不及格率逐年下降。2020 年课程的考核成绩包括以 下四个方面: 线上 MOOC 学习占比 $10 \%$ (需要完成视频学习、章节自测及期末自测等), PBL 作业成 绩 10\%, 线下作业及出勤综合 $10 \%$, 期中考试 10\%, 期末考试 60\%。据不完全统计, 2016-2020 年 的学生的不及格率基本控制在 5\%以内, 工科年级的平均分维持在 75 分以上。图 1 为 2020 年理科 实验班三个班级 91 人的课程总评成绩, 其中最高分 97 分, 最低分 47 分, 平均成绩 82.47 分。无机 化学课程的改革建设成果在 2020 年获得了省级和校级的教学成果奖。

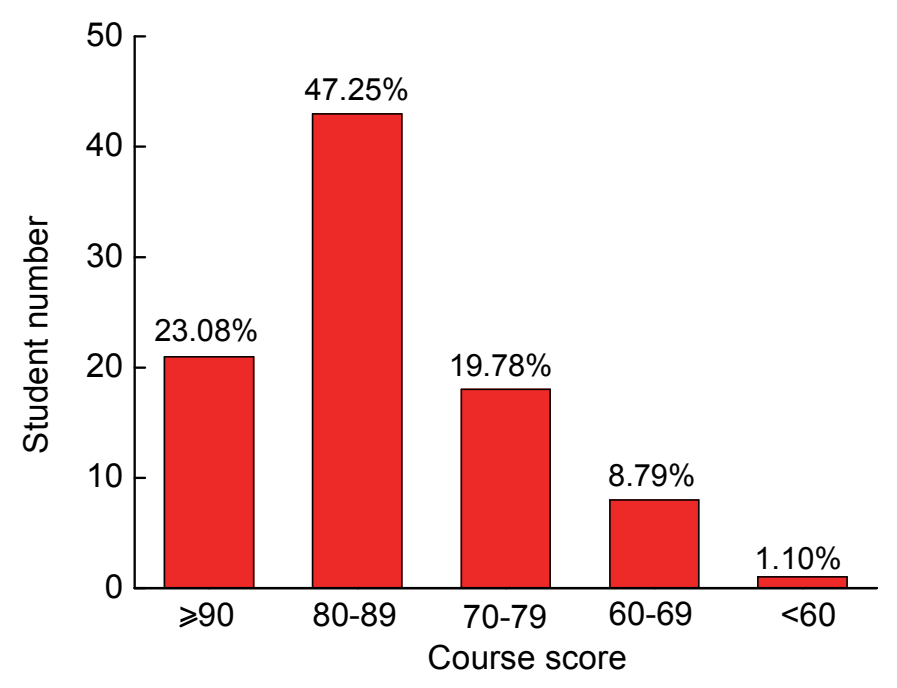

图 12020 年理科实验班 91 人的课程总评成绩分布图

无机化学在线课程起到了较好的示范作用。目前, 已经开设五期课程, 课程社会学习者所在的 地域, 包括: 辽宁、江西、河北、河北、陕西、吉林、内蒙古、江苏、山东、贵州、四川、湖北、 安徽、甘肃、云南、美国等多地。课程的全程学习者的比例较高, 特别是, 2020 年居家学习期间, 无机化学 MOOC 课程发挥了重要学习功能, 帮助学校完成了线上课程学习任务, 保证了教学质量。 
[1] 马亚鲁, 马骁飞, 田昀, 秦学, 高洪苓, 刘华姬, 鲁凡丽, 王晓东, 王兴尧, 李丹峰, 等. 大学化学, 2020, 35 (8), 48.

[2] 汪羽翎, 马荔, 谢少艾, 张卫, 魏霄, 尹屹梅, 陈虹锦. 大学化学, 2021, 36 (3), 2101047.

[3] 刘盈, 张镖, 王丽丽, 姚小丽, 张素中. 化学教育(中英文), 2020, 41 (20), 92 .

[4] 杨青林, 刘克松, 田东亮. 大学化学, 2020, 35 (9), 17.

[5] 罗映红. 高教探索, 2019, No. 12, 48.

[6] 张学新. 复旦教育论坛, 2014, 12 (5), 5 .

[7] 胡洪羽, 孙玉希, 蒋平. 化学教育(中英文), 2019, 40 (16), 60 . 